\title{
ON THE STRUCTURE OF MINIMAL SUBMANIFOLDS IN A RIEMANNIAN MANIFOLD OF NON-NEGATIVE CURVATURE
}

\author{
Gabjin Yun and Dongho Kim
}

\begin{abstract}
Let $M^{n}$ be a complete oriented non-compact minimally immersed submanifold in a complete Riemannian manifold $N^{n+p}$ of nonnegative curvature. We prove that if $M$ is super-stable, then there are no non-trivial $L^{2}$ harmonic one forms on $M$. This is a generalization of the main result in [8].
\end{abstract}

\section{Introduction}

One of the ways to study non-compact Riemannian manifolds is to use harmonic function theory in the aspect of analysis. It is well known that in order to study structures of topology and curvature of non-compact Riemannian manifolds, harmonic function theory plays an important role (cf. [6], [7] and references are therein). On the other hand, the Hodge theory shows that harmonic differential forms are important in the topology of compact Riemannian manifolds. In case of non-compact manifolds, the Hodge theory does not work well anymore. However $L^{2}$-Hodge theory remains valid in non-compact manifolds as classical Hodge theory works well in the compact case (cf. [2]).

A minimal submanifold $M$ is a critical point of the volume functional, while $M$ is said to be stable if the second variation of its volume is always non-negative for any normal deformation with compact support. Since each component function of a minimal immersion in Euclidean space is a harmonic function, any minimally immersed submanifold in Euclidean space becomes automatically non-compact by the Hopf lemma. In this direction related with stable minimal hypersurfaces, there are some known results. Miyaoka ([8]) proved that if $M$ is a complete oriented stable minimal hypersurface in a Riemannian manifold of non-negative curvature, then there are no non-trivial $L^{2}$ harmonic one forms

Received October 9, 2008.

2000 Mathematics Subject Classification. 53C21.

Key words and phrases. minimal submanifold, super-stable minimal submanifold, $L^{2}$ harmonic form.

This work was supported by the Korea Research Foundation Grant funded by the Korean Government (MOEHRD, Basic Research Promotion Fund) (KRF-2008-521-C00027). 
on $M$. The first author ([14]) proved that if $M$ is a complete oriented minimal hypersurface in Euclidean space and the total scalar curvature on $M$ is less than $n / 2$ power of the reciprocal of the Sobolev constant, then there are no non-trivial $L^{2}$ harmonic one forms on $M$. As a corollary, it is proved that such a manifold $M$ should be a hyperplane.

In higher codimensional case not hypersurfaces, one can ask whether the same property on stable minimal submanifolds holds or not. In higher codimensional case, there is a little more difficulty in analyzing $L^{2}$ harmonic differential forms because there are no known effective methods in the aspect of variation. Recently, in [9], Seo proved that if $M^{n}$ is an $n$-dimensional complete minimal submanifold in $\mathbb{R}^{n+p}$ and with flat normal bundle, then $M$ is an $n$-dimensional plane. In this paper, we deal with the existence of $L^{2}$ harmonic one forms on manifolds with higher codimension and prove that if $M^{n}$ is a complete noncompact super-stable minimal submanifold in a complete Riemannian manifold $N^{n+p}$ with non-negative curvature, then there are no non-trivial $L^{2}$ harmonic one forms on $M$. This is a generalization of known results for stable minimal hypersurfaces.

Theorem 1.1. Let $M^{n}$ be a complete oriented non-compact super-stable minimal submanifold in a complete Riemannian manifold $N^{n+p}$ of non-negative sectional curvature, then there are no non-trivial $L^{2}$ harmonic one forms on $M$.

Corollary 1.2. Let $M^{n}$ be a complete oriented non-compact super-stable minimal submanifold in $\mathbb{R}^{n+p}$, then there are no non-trivial $L^{2}$ harmonic one forms on $M$.

\section{Preliminaries}

Let $M^{n}$ be a complete non-compact minimally immersed submanifold in a complete Riemannian manifold $N^{n+p}$ of dimension $n+p$. It follows from the Gauss equation ([4]) that

$$
\overline{\operatorname{Ric}}(X, X)-\operatorname{Ric}(X, X)=\sum_{i=1}^{p}\left|A_{\nu_{i}} X\right|^{2}+\sum_{i=1}^{p}\left\langle\bar{R}\left(X, \nu_{i}\right) \nu_{i}, X\right\rangle,
$$

where $\overline{\mathrm{Ric}}$ and Ric are the Ricci curvatures of the ambient space $N$ and $M$, respectively, and $\left\{\nu_{1}, \nu_{2}, \ldots, \nu_{p}\right\}$ is a local orthonormal frame on the normal bundle of $M$ in $N$. Here $A_{\nu_{i}}$ is the second fundamental form defined by

$$
A_{\nu_{i}} X=-\left(\bar{\nabla}_{X} \nu_{i}\right)^{\top}
$$

the tangent component of the covariant derivative. In particular, in case of hypersurfaces, the equation (2.1) becomes

$$
\overline{\operatorname{Ric}}(X, X)-\operatorname{Ric}(X, X)=|A X|^{2}+\langle\bar{R}(X, \nu) \nu, X\rangle,
$$

where $\nu$ is the unit normal vector field on $M$ and $A=A_{\nu}$. 
Recall that a minimal submanifold is stable if the second variation of its volume is always non-negative for any normal variation with compact support. In case $p=1$, i.e., if $M$ is a hypersurface, this is equivalent that for any function $\phi \in C_{0}^{1}(M)$,

$$
\int_{M}|\nabla \phi|^{2}-\left(\overline{\operatorname{Ric}}(\nu, \nu)+|A|^{2}\right) \phi^{2} \geq 0
$$

where $\nu$ is an unit normal vector of $M$ and $A$ denotes the second fundamental form of $M$. However if $p>1$, the stability is not equivalent to the inequality (2.3) anymore. Instead in case $N=\mathbb{R}^{n+p}$, for example, it follows from [10] and $\overline{\mathrm{Ric}} \equiv 0$ that if $M^{n}$ is a stable minimal submanifold in a Riemannian manifold $\mathbb{R}^{n+p}$, then for any normal vector field $\nu$ and any function $\phi \in C_{0}^{1}(M)$,

$$
\left.\frac{d^{2} \mathcal{A}}{d t^{2}}\right|_{t=0} \geq \int_{M}|\nabla \phi|^{2}-\left|A_{\nu}\right|^{2} \phi^{2}
$$

where $\mathcal{A}$ is the volume functional with normal variation $\phi \nu$ and $A_{\nu}$ is defined as in the equation (2.2)

Based on the inequality (2.4), Q. Wang ([12]) introduced the concept of super-stability for minimal submanifolds in Euclidean space. Motivated by this concept, we define the notion of super-stability for minimal submanifolds in a Riemannian manifold $N^{n+p}$ as follows.

Definition 2.1. We call a minimal submanifold $M^{n}$ in $N^{n+p}$ super-stable if for any $\psi \in C_{0}^{1}(M)$ and a normal vector $\nu$ to $M$,

$$
\int_{M}|\nabla \psi|^{2}-\left(\overline{\operatorname{Ric}}(\nu, \nu)+|A|^{2}\right) \psi^{2} \geq 0
$$

Assume $p \geq 1$ and let $e_{1}, \ldots, e_{n}, \nu_{1}, \ldots, \nu_{p}$ be a (local) orthonormal frame in $N^{n+p}$ such that, restricted to $M$, the vectors $e_{1}, \ldots, e_{n}$ are tangent to $M$ and $\nu_{1}, \ldots, \nu_{p}$ are normal to $M$. Then the square norm of the second fundamental form, $|A|^{2}$, of $M$ is defined by

$$
|A|^{2}=\sum_{i=1}^{p}\left|A_{\nu_{i}}\right|^{2}=\sum_{i=1}^{p} \sum_{j=1}^{n}\left\langle A_{\nu_{i}}\left(e_{j}\right), e_{j}\right\rangle^{2} .
$$

Note that the definition of super-stability is exactly same as that of stability in case $p=1$.

Now let $\omega$ be an $L^{2}$ harmonic one form on a minimally immersed submanifold $M$ in $N^{n+p}$. This means

$$
\Delta \omega=-(d \delta+\delta d) \omega=0 \quad \text { and } \quad \int_{M} \omega \wedge * \omega=\int_{M}|\omega|^{2} d v<\infty,
$$

where $*$ denotes the Hodge star operator and $d v$ is the volume form on $M$. If $\omega$ is a harmonic one form, then its dual $\omega^{\sharp}$ is a harmonic vector field on $M$ in the following sense: if we choose a local frame $e_{1}, \ldots, e_{n}$ such that $\nabla_{e_{i}} e_{j}=0$ at a point and $\omega^{\sharp}=\omega^{i} e_{i}$, then $\nabla_{e_{i}} \omega^{j}=\nabla_{e_{j}} \omega^{i}$ and $\nabla_{e_{i}} \omega^{i}=0$ at the point. Note that $|\omega|=\left|\omega^{\sharp}\right|$. Thus from now we use confused notation for $\omega$ and $\omega^{\sharp}$. 
The following type of Bochner formula is well known:

$$
\frac{1}{2} \Delta|\omega|^{2}=|\nabla \omega|^{2}+\operatorname{Ric}(\omega, \omega) .
$$

Also we have for the Laplacian on functions

$$
\Delta|\omega|^{2}=2\left(|\omega| \Delta|\omega|+\left.|\nabla| \omega\right|^{2}\right) .
$$

Since $|\nabla| \omega|| \leq|\nabla \omega|$ by Kato's inequality, it follows from (2.5) and (2.6) that

$$
|\omega| \Delta|\omega| \geq \operatorname{Ric}(\omega, \omega) \text {. }
$$

\section{Proofs of the theorems}

In a non-compact complete Riemannian manifold, the volume growth or finiteness of the total volume plays an important role in the structure of manifolds. For instance, if $M^{n}$ is a minimal hypersurface in $\mathbb{R}^{n+1}$, then it follows from [1] that $M$ has an Euclidean volume growth. More precisely, if $x$ is a point on $M$, then

$$
\frac{\operatorname{vol}\left(B_{x}(r)\right)}{r^{n}} \geq \omega_{n}
$$

where $B_{x}(r)$ is a geodesic ball in $M$, of radius $r$ and centered at $x$, and $\omega_{n}$ is the volume of the unit ball in $\mathbb{R}^{n+1}$. In particular the volume of $M$ is infinite and this fact implies also non-existence of $L^{2}$ harmonic one forms on $M$ if $M$ is stable.

In case that the codimension is greater than one, the similar property does hold for a non-compact super-stable minimal submanifold in a Riemannian manifold of non-negative sectional curvature. A complete Riemannian manifold $M$ is said to be non-parabolic if it admits a positive Green's function. Otherwise, $M$ is said to be parabolic. $M$ is non-parabolic if and only if it admits a non-constant positive superharmonic function. Also if $M$ is non-parabolic, then for any point $x$ and a geodesic ball $B_{x}(r)$ on $M$,

$$
\int_{1}^{\infty} \frac{t}{\operatorname{vol}\left(B_{x}(t)\right)} d t<\infty .
$$

In particular, such a manifold satisfying (3.1) has infinite volume.

Lemma 3.1. Let $M^{n}$ be a complete non-compact super-stable minimal submanifold in a complete Riemannian manifold $N^{n+p}$ of non-negative sectional curvature, then $M^{n}$ has infinite volume.

Proof. It follows from (3.1) that $M$ has infinite volume if $M$ is non-parabolic. Thus we may assume that $M$ is parabolic. By using the same arguments as in [3] together with the condition that $M$ is super-stable, we can show that there exists a positive function $u$ satisfying the equation

$$
\Delta u+\left(\overline{\operatorname{Ric}}(\nu, \nu)+|A|^{2}\right) u=0
$$

on $M$. Since $\overline{\operatorname{Ric}}(\nu, \nu) \geq 0$ and $|A|^{2} \geq 0, u$ is superharmonic. Consequently it follows from parabolicity of $M$ that $u$ is constant and so we obtain that $A=0$. 
By the Gauss equation, the sectional curvature of $M, K_{M}$, is non-negative. So it follows from [13] that the volume of $M$ is infinite. Hence in any case, $M$ has infinite volume.

Next if $M$ is a complete minimal submanifold in a complete Riemannian manifold, then the Ricci curvature of $M$ is deeply related with the second fundamental form $A$ of $M$. For example if $M$ is a complete immersed minimal hypersurface in $\mathbb{R}^{n+1}$, then for any tangent vector field $X$ on $M$, it follows from the Gauss equation that $\operatorname{Ric}(X, X)=-|A X|^{2}$. In fact, P. F. Leung obtained a lower bound estimate for the Ricci curvature of submanifolds minimally immersed in Riemannian manifold of non-negative sectional curvature which is a little sharp.

Lemma $3.2([5])$. Let $M^{n}$ be a complete immersed minimal submanifold in $N^{n+p}$ of non-negative sectional curvature. Then the Ricci curvature of $M$ satisfies

$$
\operatorname{Ric}(M) \geq-\frac{n-1}{n}|A|^{2} .
$$

Now we are ready to prove our main theorem.

Theorem 3.3. Let $M^{n}$ be a complete oriented non-compact super-stable minimal submanifold in a complete Riemannian manifold $N^{n+p}$ of non-negative sectional curvature, then there are no non-trivial $L^{2}$ harmonic one forms on $M$.

Proof. Assume $\omega$ is an $L^{2}$ harmonic one form on $M$. It follows from the inequality (2.7) and Lemma 3.2 that

$$
|\omega| \Delta|\omega| \geq-\frac{n-1}{n}|\omega|^{2}|A|^{2} .
$$

Fix a point of $M$ and for $r>0$, choose a cut-off function $\psi$ satisfying the following properties:

(i) $0 \leq \psi \leq 1$,

(ii) $\left.\psi\right|_{B(r)} \equiv 1,\left.\psi\right|_{M-B(2 r)}=0$,

(iii) $|\nabla \psi| \leq \frac{2}{r}$.

Multiplying both sides by $\psi^{2}$ and integrating over $M$, we have

$$
\int_{M} \psi^{2}|\omega| \Delta|\omega| \geq-\frac{n-1}{n} \int_{M} \psi^{2}|\omega|^{2}|A|^{2} .
$$

Using integration by parts, we get

$$
\begin{aligned}
\frac{n-1}{n} \int_{M} \psi^{2}|\omega|^{2}|A|^{2} & \geq \int_{M}\left\langle\nabla\left(\psi^{2}|\omega|\right), \nabla|\omega|\right\rangle \\
& =\left.\int_{M} \psi^{2}|\nabla| \omega\right|^{2}+2 \psi|\omega|\langle\nabla \psi, \nabla|\omega|\rangle .
\end{aligned}
$$


Since $M$ is super-stable and the sectional curvature of $N$ is non-negative, we have for any $\psi \in C_{0}^{2}(M)$

$$
\int_{M}|\nabla \psi|^{2} \geq \int_{M}\left(\overline{\operatorname{Ric}}(\nu, \nu)+|A|^{2}\right) \psi^{2} \geq \int_{M}|A|^{2} \psi^{2}
$$

for any normal vector field $\nu$. Replacing $\psi$ by $\psi|\omega|$, we have

$$
\int_{M} \psi^{2}|\nabla| \omega||^{2}+|\omega|^{2}|\nabla \psi|^{2}+2 \psi|\omega|\langle\nabla|\omega|, \nabla \psi\rangle \geq \int_{M} \psi^{2}|\omega|^{2}|A|^{2}
$$

Combining (3.3) and (3.4), we obtain

$$
-\left.\frac{1}{n} \int_{M} \psi^{2}|\nabla| \omega\right|^{2}+\frac{n-1}{n} \int_{M}|\omega|^{2}|\nabla \psi|^{2}-\frac{2}{n} \int_{M} \psi|\omega|\langle\nabla|\omega|, \nabla \psi\rangle \geq 0 .
$$

Since for any positive real number $\epsilon$,

$$
\begin{aligned}
-\frac{1}{n} \int_{M} 2 \psi|\omega|\langle\nabla \psi, \nabla|\omega|\rangle & \leq \frac{1}{n} \int_{M} 2 \psi|\omega||\nabla \psi||\nabla| \omega|| \\
& \leq \frac{1}{n}\left\{\left.\int_{M} \epsilon \psi^{2}|\nabla| \omega\right|^{2}+\frac{1}{\epsilon}|\omega|^{2}|\nabla \psi|^{2}\right\}
\end{aligned}
$$

we have

$$
\left.\frac{\epsilon-1}{n} \int_{M} \psi^{2}|\nabla| \omega\right|^{2}+\frac{\epsilon(n-1)+1}{n \epsilon} \int_{M}|\omega|^{2}|\nabla \psi|^{2} \geq 0 .
$$

Choosing $0<\epsilon<1$, it follows from the properties of $\psi$ (i), (ii), (iii) that

$$
\frac{\epsilon(n-1)+1}{n \epsilon} \cdot \frac{4}{r^{2}} \int_{B(2 r)}|\omega|^{2} \geq \frac{1-\epsilon}{n} \int_{B(r)}|\nabla| \omega||^{2} .
$$

Letting $r \rightarrow \infty$, we obtain from finiteness of $L^{2}$ norm of $\omega$

$$
0 \leq \frac{1-\epsilon}{n} \int_{M}|\nabla| \omega||^{2} \leq 0 .
$$

Thus $\nabla|\omega|=0$ and so $|\omega|$ is a constant.

Since the volume of $M$ is infinity by Lemma 2.2 and $\omega$ must vanish.

Remark 3.4. As in the case of compact manifolds, the space of $L^{2}$ harmonic differential forms does satisfy the Poincaré duality. That is, the space of $L^{2}$ harmonic $k$-forms is isomorphic to the space of $L^{2}$ harmonic $(n-k)$-forms, where $n=\operatorname{dim}(M)$. Thus it follows from Theorem 3.3 that if $M^{n}$ is a complete oriented non-compact super-stable minimal submanifold in a complete Riemannian manifold $N^{n+p}$ of non-negative sectional curvature, then there are no non-trivial $L^{2}$ harmonic $(n-1)$-forms on $M$.

Acknowledgement. The authors would like to express their gratitude to the referees for suggestions and criticism. 


\section{References}

[1] H.-D. Cao, Y Shen, and S. Zhu, The structure of stable minimal hypersurfaces in $R^{n+1}$, Math. Res. Lett. 4 (1997), no. 5, 637-644.

[2] J. Dodziuk, $L^{2}$ harmonic forms on complete manifolds, in Seminar on Differential Geometry, Princeton University Press, Princeton N. J., 1982, 291-302.

[3] D. Fischer-Colbrie and R. Schoen, The structure of complete stable minimal surfaces in 3-manifolds of nonnegative scalar curvature, Comm. Pure Appl. Math. 33 (1980), no. $2,199-211$.

[4] H. B. Lawson, Lectures on Minimal Submanifolds. Vol. I, Publish or Perish, Inc., Wilmington, Del., 1980.

[5] P.-F. Leung, An estimate on the Ricci curvature of a submanifold and some applications, Proc. Amer. Math. Soc. 114 (1992), no. 4, 1051-1061.

[6] P. Li, Curvature and function theory on Riemannian manifolds, Surveys in differential geometry, 375-432, Surv. Differ. Geom., VII, Int. Press, Somerville, MA, 2000.

[7] _ Differential geometry via harmonic functions, Proceedings of the International Congress of Mathematicians, Vol. II (Beijing, 2002), 293-302, Higher Ed. Press, Beijing, 2002.

[8] R. Miyaoka, $L^{2}$ harmonic 1-forms on a complete stable minimal hypersurface, Geometry and global analysis (Sendai, 1993), 289-293, Tohoku Univ., Sendai, 1993.

[9] K. Seo, Rigidity of minimal submanifolds with flat normal bundle, Commun. Korean Math. Soc. 23 (2008), no. 3, 421-426.

[10] J. Spruck, Remarks on the stability of minimal submanifolds of $R^{n}$, Math. Z. 144 (1975), no. $2,169-174$.

[11] S. Tanno, $L^{2}$ harmonic forms and stability of minimal hypersurfaces, J. Math. Soc. Japan 48 (1996), no. 4, 761-768.

[12] Q. Wang, On minimal submanifolds in an Euclidean space, Math. Nachr. 261/262 (2003), 176-180.

[13] S.-T. Yau, Some function-theoretic properties of complete Riemannian manifold and their applications to geometry, Indiana Univ. Math. J. 25 (1976), no. 7, 659-670.

[14] G. Yun, Total scalar curvature and $L^{2}$ harmonic 1-forms on a minimal hypersurface in Euclidean space, Geom. Dedicata 89 (2002), 135-141.

GABJIN YUN

Department of Mathematics

MYONG Ji UNIVERSITY

KyungGi 449-728, KoreA

E-mail address: gabjin@mju.ac.kr

DONGHO KIM

Department of Mathematics

MYONG Ji UNIVERSITY

KYUNGGI 449-728, KoreA

E-mail address: mjukdh98@mju.ac.kr 